\section{Darmkrebs: DNA im Blut als Prognosemarker}

\author{
Bei Patienten mit metastasiertem kolorektalem Karzinom ( $m C R C$ ) lässt \\ sich Tumor-DNA im Blut nachweisen. Nun wurde bei Patienten unter \\ Tyrosinkinaseinhibition untersucht, ob zirkulierende Tumor-DNA von \\ prognostischem Wert ist.
}

V on über 500 Patienten mit mCRC, die in der Phase-III-Studie CORRECT behandelt wurden, wurde sowohl die Tumor-DNA aus dem Plasma isoliert als auch die Konzentration von 15 verschiedenen Proteinen gemessen, darunter vaskulärer endothelialer Wachstumsfaktor (VEGF), Makrophagen-CSF, TIE-1 und Von-Willebrand-Faktor. Auf der Basis des Mutationsstatus, der Menge an zirkulierender DNA und der Proteinkonzentrationen im Blut wurde in der retrospektiven Studie die Korrelation mit Gesamt- und progressionsfreiem Überleben überprüft. Für die Mutationsanalyse wurden die Gene KRAS, BRAF und PIK3CA zu Beginn der Studie unter die Lupe genommen. Wie aus der Auswertung hervorgeht, haben Mutationen in der zirkulierenden Tumor-DNA prognostischen Wert. Während die Hazard Ratio (HR) für das Gesamtüberleben im gesamten Kollektiv unter Regorafenib im Vergleich zu Placebo bei 0,77 (95\%-Konfidenzintervall [95\%-KI] 0,64-0,94) lag, ermittelten die Wissenschaftler für Patienten mit WildtypKRAS einen niedrigeren Wert (HR 0,67, $95 \%$-KI 0,41-1,08). Die Wahrscheinlichkeit, mit dem Kinasehemmer zu überleben, war hier somit größer. Bei Patienten mit KRAS-Mutation lag die HR etwas höher (HR 0,81, 95\%-KI 0,61-1,09), der Unterschied jedoch nicht signifikant.

Die HR-Werte im Zusammenhang mit dem Gesamtüberleben nach Analyse des PIK3CA-Gens lagen bei 0,75 (nicht mutiert) und 0,84 (mutiert). Einen noch deutlicheren Vorteil für die Behandlung mit Regorafenib ergaben die Resultate der Genanalysen beim KRASund PIK3CA-Gen im Zusammenhang mit dem progressionsfreien Überleben.

Nicht unerwartet korreliert noch mehr der Nachweis frei zirkulierender Tumor-DNA mit dem Gesamtüberleben. Die Wahrscheinlichkeit zu überleben ist der Studie zufolge bei hohen DNA-Konzentrationen im Plasma signifikant geringer, unter Placebo wie unter Regorafenib (HR mit Regorafenib: 0,34).

Fazit: Das Interesse an der diagnostischen Verwertung zirkulierender $\mathrm{Tu}$ mor-DNA ist deutlich gestiegen. Deren Menge zu Therapiebeginn eignet sich bei Patienten mit mCRC unter einem Tyrosinkinasehemmer offenbar als Prognosemarker.

Peter Leiner

Tabernero J et al. Analysis of circulating DNA and protein biomarkers to predict the clinical activity of regorafenib and assess prognosis in patients with metastatic colorectal cancer: a retrospective, exploratory analysis of the CORRECT trial. Lancet Oncol. 2015;16(8):937-48.

\title{
Nach Darmkrebsoperation: Zeitfenster bis zur Chemotherapie
}

\section{Für frisch operierte Patienten mit Darmkrebs (CRC) im Stadium III gibt es offenbar ein Zeitfenster, in dem sie sich ohne besonderes Risiko bis zum Beginn einer adjuvanten Chemotherapie erholen können.}

\footnotetext{
n der niederländischen Studie wurden 12.654 Patienten, bei denen zwischen 2008 und 2013 ein Kolonkarzinom im Stadium III (d. h. mit positiven Lymphknoten, aber ohne Fernmetastasen) diagnostiziert und der Tumor daraufhin reseziert worden war, beobachtet. $7.755 \mathrm{~Pa}-$ tienten erhielten im Anschluss an die Resektion eine adjuvante Chemotherapie. Die Mehrheit (69\%) startete damit zwischen 5-8 Wochen postoperativ. $17 \%$ erhielten die erste Dosis bereits innerhalb von vier Wochen, $14 \%$ erst ab der 8. Woche. Bei 165 Patienten (2\%) verzögerte sich der Therapiestart bis zur 13. - 16. Woche postoperativ. Im Median
}

wurden die Patienten über 60 Monate nachbeobachtet. Die Chance, diesen Zeitraum zu überleben, lag bei Patienten mit einem Chemotherapie-Beginn zwischen 5 und 6 Wochen nach Operation bei $76 \%$, bei Beginn 7-8 Wochen postoperativ bei $72 \%$. Ab der 9 . Woche sanken die 5-Jahres-Überlebensraten jedoch deutlich: auf $64 \%$ bei Beginn in Woche $9-10$, auf $61 \%$ in Woche $11-12$ und auf $54 \%$ bei noch späterem Start. Damit war das Risiko, innerhalb des Beobachtungszeitraums zu versterben, bei einem Therapiestart erst nach 9-10 Wochen um $40 \%$, nach 11-12 Wochen um $30 \%$ und nach $13-16$ Wochen um $70 \%$ erhöht (jeweils im Vergleich zum Beginn binnen 8 Wochen nach Operation). Noch vor Ablauf eines Monats mit der Chemo anzufangen, schien allerdings auch keinen Vorteil zu bringen; in diesem Fall betrug die Überlebenswahrscheinlichkeit $75 \%$ - kein signifikanter Unterschied also zu einem Beginn inner- halb des Zeitfensters zwischen 5 und 8 Wochen.

Vor allem folgende Faktoren hatten Einfluss auf das Timing: eine Anastomosen-Insuffizienz nach Operation, ein $\mathrm{Al}$ ter über 75 Jahre, eine notfallmäßig vorgenommene Resektion oder eine Verlegung in ein anderes Krankenhaus. Alle waren mit einem Therapiebeginn nach der 8. Woche verknüpft (Odds Ratios: $8,1 ; 1,6 ; 1,8 ; 1,9)$.

Fazit: Nach Resektion eines CRC im Stadium III kann man offenbar ohne besonderes Risiko bis zu 8 Wochen abwarten, bevor man mit einer adjuvanten Chemotherapie startet. Die Patienten können sich in dieser Zeit von der Op. erholen und Kraft für den nächsten Therapieschritt sammeln. Elke Oberhofer

Bos ACRK et al. Timing of adjuvant chemotherapy and its relation to survival among patients with stage III colon cancer. Eur J Cancer. 2015 Sep 7. [Epub ahead of print]. 